\title{
AVALIAÇÃO DE RISCO NO PROCESSO DE INTERNACIONALIZAÇÃO DA NBA NO BRASIL
}

\author{
Amaury Floriano Portugal Neto ${ }^{1}$, Sergio Henrique Arruda Cavalcante Forte \\ Centro Universitário INTA, Sobral, Ceará, (Brasil) \\ Universidade de Fortaleza - INIFOR, Fortaleza, Ceará, (Brasil)
}

\section{DETALHES DO ARTIGO}

Recebido: 16 de novembro de 2018

Aceito: 05 de setembro de 2019

Disponível online: 01 de jan. de 2020

Sistema de revisão "Double blind review"

Editor Científico

Ilan Avrichir

\section{Palavras-chaves:}

Processo de internacionalização

Avaliação de risco

Gestão do esporte

Basquete. NBA

\begin{abstract}
RESUMO
Objetivo: Avaliar os riscos envolvidos no processo de internacionalização da NBA no Brasil no seu período inicial, 2012-2015 e uma projeção sobre o seu futuro 5 anos à frente, 2020.

Método: A metodologia compreendeu seis fases, envolvendo pesquisa bibliográfica, consulta a peritos para levantamento de dimensões, avaliação e refinamento de variáveis relevantes, permitindo a formatação do Índice de Avaliação de Risco da NBA (IARnba) e sua aplicação à luz do modelo de Figueira de Lemos, Johanson e Vahlne (2011) na análise sobre a internacionalização da NBA, tendo as variáveis de (C) comprometimento e (I) Incerteza interpretados.

Principais resultados: $O$ estudo demonstrou que durante os dois períodos estudados o risco de internacionalização da NBA no Brasil tende a diminuir, apesar dos níveis de incerteza se manter em pequena queda. O crescente nível de conhecimento do mercado brasileiro seguido de investimentos que apontam um maior comprometimento corrobora com essa visão.

Relevância/originalidade: Sua relevância se dá em razão de seu ineditismo em retratar o foco na internacionalização de ligas esportivas em países emergentes e de economia instáveis como o Brasil.

Contribuições teóricas/metodológicas: Sua contribuição com a literatura ao abordar a temática do sports business à luz da teoria da internacionalização da firma, retratando a busca de mercados internacionais de ligas esportivas mundiais e por demonstrar e avaliar os riscos destes movimentos por meio de um índice.
\end{abstract}

\section{Introdução}

Empresas de serviços globais, especialmente serviços profissionais, tem se tornado um dos setores de maior crescimento da economia mundial e este crescimento fomenta a necessidade de pesquisas na área de serviços profissionais internacionais (Javalgi et al., 2011). Dentro deste contexto, destaca-se a área de esportes, que compreende uma grande parte da economia global e sua contribuição impacta várias outras áreas (Ratten, 2011a).

No status de fenômeno global que o esporte adquiriu durante todo o século 20 , intensificado no século 21 , à luz da globalização e novas mídias e tecnologias, houve 0 desenvolvimento da internacionalização do esporte em geral como fenômeno, fato observado quando organizações esportivas relevantes como a NASCAR (automobilismo), UFC (lutas), Premier League (futebol) e a NBA (basquete) oferecem seus produtos e serviços para fãs fora de seus países de origem (Chadwick, 2009; Ratten, 2018).

A NBA, liga profissional de basquete dos Estados Unidos, é a organização esportiva mais bem sucedida na área internacionalização do esporte. Fruto de sua visão focada no mercado internacional, ancoradas em campanhas publicitárias mundiais combinadas com eventos midiáticos diversos (responsabilidade social, clínicas e atrativos para fãs) (Eisenberg, 2003; Euchner, 2008; Kian, 2009; Proni, 1998).

O Brasil, sempre se mostrou um mercado receptivo à NBA. Indicadores de consumo de serviços e produtos da liga no país são impulsionados com o constante aumento do número de jogadores em suas franquias, fato ampliado pela criação da NBA Brasil em 2012. Desde então, o modus operandi tradicional foi ampliado, com o aumento em presença em mídia aberta e a realização de jogos anuais entre suas 
franquias em solo brasileiro, o NBA Global Games Rio. (Balassiano, 2015; NBA, 2018).

Neste fenômeno observa-se também barreiras de entrada relevantes. Economia em recessão desde 2015, incertezas políticas no período, domínio esportivo e midiático do futebol, péssima estrutura esportiva e desinteresse do brasileiro pela prática do esporte são barreiras de entrada ao processo de internacionalização da NBA no Brasil (Deloitte Brasil, 2010; Pluri Consultoria, 2014; Portugal Neto \& Forte, 2015; Sport+Markt,2011).

Estas barreiras somadas ao investimentos em ativos financeiros, humanos e mercadológicos realizados pela NBA Brasil, configuram um cenário em que o risco se faz presente a todo momento. $\mathrm{Na}$ literatura de Internacionalização o risco de um mercado existente se define pelo produto do comprometimento em recursos despendidos neste mercado pelo grau de incerteza que se tem a respeito neste mercado (Figueira-de-lemos, Johanson, \& Vahlne, 2011).

A internacionalização da NBA no Brasil, seguida de uma piora considerável nas condições institucionais do país, leva a necessidade de se avaliar os riscos envolvidos neste processo e que diante deste contexto pergunta-se: Como avaliar os riscos do processo de internacionalização da NBA no Brasil?

Assim, esta pesquisa tem como objetivo geral avaliar os riscos do processo de internacionalização da NBA no Brasil e como objetivos específicos: a elaboração de um índice de avaliação de risco de internacionalização da NBA (IARnba) no Brasil; e a mensuração do risco de internacionalização da NBA no Brasil em seu período inicial 2012-2015 e sua evolução com uma projeção em cinco anos, para 2020. A metodologia adotada inicialmente se empenhou na busca de possíveis variáveis e na definição de um método de avaliação de risco. Para tanto uma combinação de levantamento bibliográfico, revisão de literatura e entrevistas exploratórias com especialistas foram adotadas para o refinamento e a seleção dessas variáveis coletadas. Em seguida o processo avançou com aplicação de questionários a 25 peritos com atribuição de pesos e notas as variáveis que com análise dos resultados sofreu um refinamento e corte final chegando as condições para a formulação do índice IARnba.

Este trabalho torna-se relevante por contribuir em três abordagens: metodológica, empírica e de contribuição gerencial e de ordem prática. Em uma abordagem metodológica prevê o desenvolvimento de um Índice de Avaliação de Risco da NBA (IARnba), mesclando o Risco de Internacionalização de Figueira de Lemos, Johanson, e Vahlne (2011) à luz da literatura da gestão de esportes de Chadwick (2009) e Ratten (2011a), em um índice que pode ser aplicado a avaliação de risco de forma constante e por diversas instituições esportivas no mercado Brasileiro e em diversos mercados a que se pretende avaliar.

Em uma abordagem empírica se justifica em razão de seu ineditismo. O foco na internacionalização de ligas esportivas é relevante uma vez que desde a realização da Copa do Mundo de Futebol e Olimpíadas, o Brasil consolidou-se como mercado para eventos esportivos mundiais, com o mercado aberto para outras ligas esportivas como UFC (lutas), NFL (futebol americano) e WSL (Surf).

Na contribuição gerencial e de ordem prática, a pesquisa contribui também para a análise do estágio atual de internacionalização da NBA no Brasil, tomando por base a opinião de peritos de várias instituições ligadas ao Basquete como atuais e exjogadores, jornalistas, dirigentes e executivos das diversas instituições que representam o esporte no Brasil, incluindo a NBA, mapeando todos os movimentos dentro desse processo nos últimos 20 anos e apontando para a sua situação futura em 2020.

\section{Internacionalização no Basquete e as Ligas Esportivas Profissionais Globais}

Proni (1998) demonstra que a partir dos anos de 1960, empresas, instituições e eventos esportivos já buscavam soluções na busca de oportunidades em mercados internacionais. Este processo de internacionalização fez com que o esporte tenha evoluído para uma importante atividade econômica geradora de riqueza (Goldman \& Johns, 2009). Produtos e serviços esportivos vem sendo internacionalizados por intermédio de sua comercialização e promoção em todo o mundo (Bauer; Sauer; \& Schmitt, 2005).

Chadwick (2009) e Ratten (2011a) têm contribuído ativamente com trabalhos na dedicados a indústria do esporte, demonstrando o atual panorama das pesquisas acadêmicas relacionadas a esta indústria, com ênfase ao estágio atual e tendências futuras, tendo Ratten (2011a) aprofundado na internacionalização do esporte. 
Dentro da área de Internacionalização do esporte, Chadwick (2009) afirma que os possíveis desafios a serem trabalhados são: Estratégias de entrada em mercados; exportação e entrega de produtos culturais no exterior; Adaptação a exigências de mercado locais e questões de monitoramento e controle, mostrados de forma resumida na figura 1.

LISTA DE FIGURAS E TABELAS

\begin{tabular}{|c|l|l|}
\hline \multicolumn{1}{|c|}{ Área } & \multicolumn{1}{|c|}{ Foco de Assuntos Emergentes } & \multicolumn{1}{c|}{ Desafios Gerenciais impostos } \\
\hline \multirow{3}{*}{ Internacionalização } & $\begin{array}{l}\text { Desenvolvimento de atividade } \\
\text { internacionais/globais; }\end{array}$ & $\begin{array}{l}\text { Considerações sobre estratégias de } \\
\text { modos de entrada; }\end{array}$ \\
\cline { 2 - 4 } & Influência da Internacionalização; & $\begin{array}{l}\text { Assuntos pertinentes à exportação ao } \\
\text { exterior e entrega de produtos culturais; }\end{array}$ \\
\cline { 2 - 4 } & $\begin{array}{l}\text { Relacionamento com consumidores } \\
\text { efornecedores globais. }\end{array}$ & $\begin{array}{l}\text { Adaptação de produtos globais a } \\
\text { requisitos locais; monitoramento e } \\
\text { controle de ações. }\end{array}$ \\
\hline
\end{tabular}

Figura 1. Sumário de áreas de pesquisas potenciais na Internacionalização do Esporte Fonte: Chadwick, 2009

Ratten (2011a) mostra as possíveis subáreas a serem estudadas dentro da área específica de Internacionalização do Esporte. Essas subáreas influenciaram a busca e formação de variáveis utilizadas nesta pesquisa, aplicadas as ações da NBA no Brasil, liga esportiva que é objeto da pesquisa.

Fatores como branding no esporte, desenvolvimento regional do esporte e marketing esportivo internacional, retiradas do trabalho de Ratten (2011a), congregam e explicam boa parte das ações de empresas e marcas em seus mercados e abastecem diretamente a base teórica para os estudos de internacionalização do esporte utilizadas neste trabalho. As ligas esportivas tem papel fundamental nestas ações.

As ligas esportivas profissionais globais têm por característica o foco na internacionalização de suas marcas (Balmer \& Gray, 2003). Os gestores dessas ligas concentram-se em questões globais, tais como as condições econômicas, novas tecnologias, calendário internacional de jogos e direitos de transmissão (Howard \& Crompton, 2004). De acordo com Ratten (2011a), as ligas profissionais esportivas americanas de futebol americano, National Football League (NFL) e de baquete, National Basketball Association (NBA) são as ligas mais conhecidas do mundo, sendo a NBA a mais internacionalizada delas.

Proni (1998) mostra que ações de internacionalização da NBA são constantes no mundo desde as olimpíadas de Barcelona em 1992. Tendo seus jogadores atuando como autênticos garotos propagandas na abertura de mercados. Portugal Neto \& Forte, (2015) afirmam que durante toda a década de 2000 a NBA abriu vários escritórios pelo mundo, à medida que suas franquias iam recrutando jogadores de outros países. Tendo esta atuação caracterizada pela busca por contratos de tv neste países, além de realização de eventos e venda de merchandising dos times e jogadores nativos. Lee (2007) coloca como marco estratégico a contratação de Yao Ming, primeiro atleta chinês a jogar na Liga de Basquete americana, abrindo assim a China e seu imenso mercado consumidor.

A Forbes (2018), em sua pesquisa anual afirma que a NBA, faturou em 2018, 8 bilhões de dólares e suas franquias juntas têm valor de mercado de 40 bilhões de dólares, sendo que $30 \%$ deste valor advêm de mercados internacionais. Ainda a Forbes (2018) afirma que o faturamento da liga era de 2,5 bilhões de dólares em 2001, sendo este valor 3 vezes superior em 2018.

Porém não há estudos que associem diretamente essas ações de ligas esportivas globais em busca de mercados às teorias de internacionalização da firma. E este estudo não objetiva classificar essas ações nas teorias conhecidas e existentes.

Optou-se pelo modelo gradual da "Escola de Upsalla" como teoria base para o desenvolvimento do estudo, algo que será observado na próxima seção.

\section{Avaliação de Risco no Processo de Internacionalização no Modelo Gradual da Escola de Uppsala}

O Processo de internacionalização de empresas pode ser definida como um processo crescente e continuado de envolvimento das operações de uma empresa com países fora de sua base de origem (Goulart, Brasil, \& Arruda 1996). 
As teorias do processo de internacionalização são agrupadas em duas categorias: abordagens baseadas em decisões econômicas, que são orientadas para a otimização de lucros e retornos financeiros e abordagens comportamentais evolutivas, baseado na evolução gradual e comportamental da empresa e na procura da redução de riscos com maiores retornos na medida que se acumula experiência no exterior (Geleilate, 2011; Raisanen, 2003).

Dentro das teorias baseado na evolução gradual e comportamental da empresa, detaca-se a teoria da Escola de Upsalla. O modelo, baseado em observações empíricas de estudos de negócios internacionais, mostra que empresas suecas frequentemente desenvolviam suas operações internacionais em pequenos passos. (JOHANSON; VAHLNE, 2009).

Fleury e Fleury (2007) afirma que a internacionalização vem de forma gradual (incremental) mediante um processo de aprendizado. É o modelo em que as empresas gradualmente adotam um processo sequencial de internacionalização movendo-se em direção a países mais próximos a fim de evitar incertezas e minimizar riscos.

Existem alguns tipos de riscos já estudados no processo de internacionalização: Empreendedorismo (Lumpkin \& Dess, 1996), Processo de Internacionalização (Johanson \& Vahlne, 2009) e (Dunning, 2006), Percepção de Risco Gerencial em decisões de modos de entrada internacionais (Forlani, Parthasaranthy, \& Keaveney 2007), Modos de Entrada (Sharma \& Erramili 2004), Riscos de Negociação (McDougall, 1989), (Williamson, 1995), (Child \& Rodrigues, 2007), Internalização de Mercados (Buckley \& Casson, 1976).

Para Dikmen e Birgonul (2006) a avaliação do risco em projetos internacionais é uma tarefa bastante complicada devido à sensibilidade relativa aos riscos de cada país e do projeto em si. Quem toma as decisões tem bastante dificuldade em pesar a relação oportunidade versus risco, sendo que as oportunidades podem ser medidas segundo o que se espera do desempenho de um projeto.

Dentro da literatura o modelo de Uppsala de Johanson e Vahlne (1977), trabalha o risco e a necessidade de minimizá-lo como fator preponderante no processo de internacionalização das empresas. A internacionalização gradual iniciada com a exportação favorece a aprendizagem e mitiga os riscos. Quando o empresário decide fazer investimentos diretos, a aquisição de negócios em mercados estrangeiros é uma opção para reduzir o risco por adquirir a propriedade em pleno funcionamento (Schweizer et al., 2010).

Figueira-de-lemos, Johanson e Vahlne (2011), revisitam esta teoria inicial da escola de Uppsala propondo um construto para avaliar o risco do processo de internacionalização da firma em até sete possíveis estágios a partir da visão das variáveis de Comprometimento e Incerteza.

O pressuposto básico do modelo de Uppsala, o mecanismo de internacionalização, é analiticamente apoiado por uma fórmula matemática que relaciona Risco, Comprometimento e Incerteza (Figueira de Lemos; Johanson; \& Vahlne, 2011).

A variável Risco pode ser definida pela expressão matemática $\mathrm{Ri}=\mathrm{Ci} \times \mathrm{Ui}$, onde assume que $\mathrm{Ri}, \mathrm{a}$ situação de risco do mercado existente é o produto do comprometimento do mercado existente Ci pelo grau de incerteza do mercado existente Ui (Johanson \& Vahlne, 1977).

Johanson e Vahlne (1977) afirmam que a forma mais fácil de definir comprometimento é usando sua faceta quantitativa o que pode ser relacionado ao investimento em marketing, gestão, pessoal e outros recursos.

Figueira de Lemos, Johanson e Vahlne (2011) atualizam que a dimensão incerteza está correlacionada com conhecimento. Quanto mais conhecimento a firma adquirir, menores serão as incertezas a respeito do mercado e assim pode-se concluir que a causa da "incerteza contingente" é a falta de conhecimento.

A fim de melhor demonstrar esta relação das variáveis na equação, os autores criaram um framework onde pode-se analisar o estágio em que uma empresa está no processo de internacionalização, baseado nas variáveis de (C) comprometimento e (I) Incerteza a serem presentadas e interpretados. Veja-se a figura 2. 


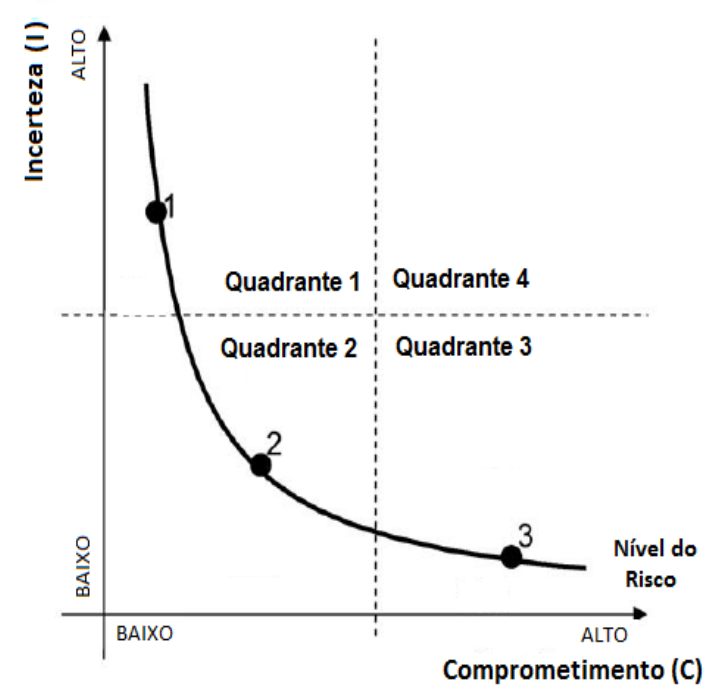

Figura 2. Internacionalização e quadrantes de decisão Fonte: De Figueira-de-lemos; Johanson e Vahlne (2011)

Na Figura 2, observa-se uma curva que caracteriza a internacionalização de uma firma num mercado estável. A medida que a curva desce do ponto 1 ao ponto 3, a empresa diminui seu nível de incerteza em relação ao mercado, de forma natural seu comprometimento com o mercado aumenta o que consequentemente diminui o risco de se atuar naquele mercado (Figueira de Lemos; Johanson; \& Vahlne, 2011).

Esta curva se aplica e é interpretada num mercado considerado estável. Caso ocorram instabilidades neste mesmo mercado, que é o caso do Brasil, por exemplo, existem sete possíveis hipóteses ou caminhos que podem ser considerados pelas empresas dentro dos quatro quadrantes apontados pelos autores com a aplicação dos índices, as quais temos as possíveis interpretações abaixo:

Quadrante 1: Alta incerteza e Baixo Comprometimento: Este quadrante trabalha a fase inicial de internacionalização. O conhecimento da firma em relação ao mercado é pequena como também o comprometimento ainda é pequeno. As posições neste quadrante vão refletir no futuro se a empresa deve esperar adquirir mais conhecimento sobre o mercado ou sair do mesmo. Quadrante 2: Baixa Incerteza e Baixo Comprometimento: Neste quadrante a empresa já possui um pouco mais de conhecimento sobre os mercados o que dá segurança para o estabelecimento de um escritório de vendas. As posições aqui podem refletir no futuro uma evolução com aquisição de ativos tangíveis mais fortes como uma fábrica local ou se houver possíveis problemas à vista, a empresa pode esperar continuando o aprendizado do mercado fortalecendo essa relação saudável.

Quadrante 3: Pequena Incerteza e Alto Comprometimento: Neste quadrante a realidade de alto comprometimento impera estando a empresa estabelecida de forma plena no mercado exterior em termos comerciais e industriais. A empresa conhece o mercado e se sente segura nele. As posições podem refletir no futuro um comprometimento da empresa ainda maior em termos de ativos tangíveis dependendo se o cenário se mostra ainda mais promissor, ou não, apenas concentrando os níveis atuais de comprometimento.

Quadrante 4: Alta incerteza e Alto comprometimento: Neste quadrante temos uma situação extrema caracterizada por um aumento grande no grau de incerteza caracterizado por um conhecimento obsoleto a respeito do mercado local, onde já se tem uma alto grau de comprometimento que não permite uma eventual volta para o pais sede sem sofrimento. Nesta situação as posições aqui mostraria que num possível aumento do risco o mais recomendável seria a empresa reforçar os relacionamentos já conquistados e esperar.

\section{METODOLOGIA}

Esta pesquisa se define como de abordagem epistemológica positivista; exploratória e foi concebida como uma pesquisa quantitativa. A 
estratégia de pesquisa escolhida foi o método survey, e a postura metodológica uma vez que se pretende produzir descrições quantitativas da população pesquisada (Freitas et al., 2000).

A primeira fase foi caraterizada pela realização de um levantamento bibliográfico realizado no período de janeiro de 2015 a julho de 2016, para coleta de dados e levantamento de variáveis, trabalhando autores, bases de dados em plataformas e banco de dados virtuais como CAPES e ESBCOhost, contemplando três temáticas: Processo de Internacionalização, Avaliação de Risco e Avaliação de Risco no Processo de Internacionalização.

A segunda fase caracterizou-se pela definição do método de avaliação de risco por Figueira de Lemos, Johanson e Vahlne (2011), atualização proveniente da literatura do Processo de Internacionalização da Firma, proposto por Johanson e Vahlne (1977) na teoria clássica de Uppsala que considera duas dimensões e suas possíveis variáveis dentro de um processo de avaliação de risco: Incerteza (Uncertainty) e Comprometimento (Commitment).

A terceira fase caracterizou-se pela realização de entrevistas exploratórias com quatro peritos na área do objeto do estudo para validação de informações e definição de variáveis. O perfil desses especialistas foi definido pelo nível de conhecimento em competências diferentes do objeto, procurando aglutinar um time de atores que por participarem de forma ativa no universo estudado poderiam contribuir de forma relevante.

$\mathrm{Na}$ quarta fase aconteceu o processo de refinamento desses dados. Trabalhou-se o processo de seleção e classificação desses dados coletados e seu refinamento teórico, considerando os trabalhos de Chadwick (2009) e Ratten (2011a) e a consequente redução das variáveis para o número de 20, na tabela 1.

A quinta fase do trabalho, foi caracterizada pela confecção e aplicação de questionários à peritos, Inicialmente pensou-se numa universo de 25 peritos, pois para dar-se relevância ao estudo, precisaríamos abranger todas as instituições e stakeholders que de fato fizesse presente na cadeia produtiva do basquete no Brasil, esses peritos são profissionais integrantes ativos da cadeia produtiva do basquete nacional, instituições e esferas de organização do Basquete Brasileiro, localizados nas cidades de Fortaleza-CE, Rio de Janeiro-RJ, São Paulo-SP, Curitiba-PR, Franca-SP, Bauru-SP, Miami-FL, com reconhecida capacidade de análise dos acontecimentos dentro do objeto pesquisado. Com a utilização do aplicativo Survey Monkey para a disseminação e aplicação, no período de agosto a novembro de 2016.

O questionário enviado aos peritos continham quatro solicitações - inicialmente a atribuição de pesos e notas nos dois períodos desta pesquisa, 2012-2015 e 2020, considerando a pertinência de cada variável com a finalidade de contribuir para a construção de um Índice de Avaliação de Risco (IAR). No questionário, foram listadas as variáveis identificadas nas fases anteriores, dimensionadas pelos respondentes, que foram avaliadas mediante 0 uso de uma escala Likert de 1 a 5 atribuindo a pesos: 1 =irrelevante; 2 = pouco pertinente; $3=$ medianamente pertinente; 4 = pertinente; 5 = muito pertinente.

Na sexta e última fase da pesquisa criou-se o índice larNBA e classificou-se o risco de internacionalização da NBA no Brasil nos períodos já citados baseado no framework desenvolvido por Figueira de Lemos, Johanson e Vahlne (2011), visando analisar o estágio em que uma empresa está no processo de internacionalização, baseado nas variáveis de (C) comprometimento e (I) Incerteza colhidos no questionário e assim interpretados. O processo acima vem todo ele demostrado na próxima seção.

O recorte de tempo analisado para classificar o Risco de Internacionalização da NBA no Brasil, foi em seu período inicial 2012-2015 e numa projeção de sua evolução numa projeção em 5 anos, para 2020. 
Tabela 1

Variáveis finais do Processo de confecção do Índice IARnba

\begin{tabular}{|c|c|c|}
\hline Dimensões & $\#$ & Variáveis Finais \\
\hline \multirow{10}{*}{ 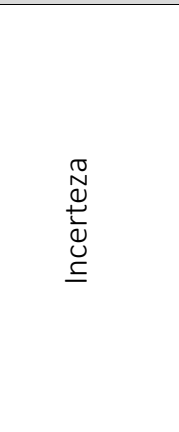 } & 1. & Economia Brasileira \\
\hline & 2. & Desenvolvimento do Esporte no Brasil \\
\hline & 3. & Monopólio Esportivo e Econômico - Futebol no Brasil \\
\hline & 4. & Cultura Esportiva na Sociedade Brasileira \\
\hline & 5. & Popularidade do Basquete no Brasil \\
\hline & 6. & Ídolos Nacionais do Basquete Brasileiro \\
\hline & 7. & Infraestrutura Esportiva no Brasil - Arenas \\
\hline & 8. & Transmissões de jogos de Basquete na TV Aberta \\
\hline & 9. & Calendário Nacional Anual de jogos de Basquete \\
\hline & 10. & Estrutura de Base para a Formação de Novos Jogadores \\
\hline \multirow{10}{*}{ 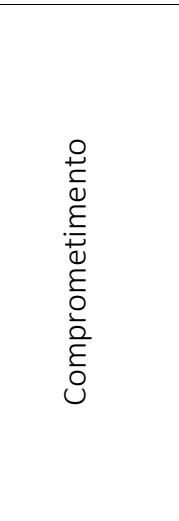 } & 11. & Criação de Escritório Comercial no Brasil \\
\hline & 12. & Realização de Jogos de Pré-temporada entre franquias da NBA no Brasil \\
\hline & 13. & Realização de jogos entre equipe brasileira e franquia americana nos EUA \\
\hline & 14. & Realização de jogo entre equipe brasileira e franquia americana no Brasil \\
\hline & 15. & Parceria Formal de 3 anos com o NBB \\
\hline & 16. & Tradução do site da NBA.com para o português \\
\hline & 17. & $\begin{array}{l}\text { Acordos de tv que ampliam o número de jogos transmitidos em TV fechada de } 2 \text { pra } 5 \\
\text { semanalmente }\end{array}$ \\
\hline & 18. & Abertura de Loja Virtual de produtos oficiais em Português \\
\hline & 19. & $\begin{array}{l}\text { Realização de pelo menos um evento voltado ao consumidor no Brasil de forma regular desde } \\
2003\end{array}$ \\
\hline & 20. & Criação de Perfis em Redes Sociais e de conteúdo específico para o público brasileiro. \\
\hline
\end{tabular}

Nota. As fontes foram pesquisas bibliográficas e entrevistas exploratórias realizadas pelos próprios autores (2015).

\section{Análise e Discussão dos Resultados}

As entrevistas estruturadas feito com peritos na Fase 3 deste trabalho, foi realizado com o objetivo de se conseguir variáveis para compor o questionário dentro das dimensões já definidas. A contribuição dos peritos foi determinante atribuindo relevância ao trabalho e validando as variáveis e dimensões escolhidas. Um resumo das conclusões estão expostas na tabela abaixo.

Tabela 2

Contribuição dos peritos na seleção e refinamento de variáveis

\begin{tabular}{|c|c|c|}
\hline Questões & Dimensão & Variáveis \\
\hline \multirow{3}{*}{ Questões 04 e 07} & \multirow{3}{*}{ INCERTEZA } & Cultura Esportiva na Sociedade Brasileira \\
\hline & & Monopólio Esportivo e Econômico - Futebol no Brasil \\
\hline & & Economia e Política Brasileira \\
\hline Questão 05 & INCERTEZA & Infraestrutura Esportiva no Brasil - Arenas \\
\hline \multirow{7}{*}{ Questão 09} & \multirow{7}{*}{ COMPROMETIMENTO } & Parceria Formal de 3 anos com o NBB \\
\hline & & $\begin{array}{l}\text { Realização de Jogos de Pré-temporada entre franquias da NBA no } \\
\text { Brasil }\end{array}$ \\
\hline & & $\begin{array}{l}\text { Realização de jogos entre equipe brasileira e franquia americana } \\
\text { nos EUA }\end{array}$ \\
\hline & & $\begin{array}{l}\text { Realização de jogos entre equipe brasileira e franquia americana } \\
\text { no Brasil }\end{array}$ \\
\hline & & Abertura de Loja Virtual de produtos oficiais em Português \\
\hline & & $\begin{array}{l}\text { Criação de Perfis em Redes Sociais e de conteúdo específico para o } \\
\text { público brasileiro. }\end{array}$ \\
\hline & & Tradução do site da NBA.com para o português \\
\hline \multirow{3}{*}{ Questão 10} & \multirow[b]{2}{*}{ COMPROMETIMENTO } & Criação de Escritório Comercial no Brasil \\
\hline & & $\begin{array}{l}\text { Realização de pelo menos um evento voltado ao consumidor no } \\
\text { Brasil de forma regular desde } 2003\end{array}$ \\
\hline & INCERTEZA & Ídolos Nacionais do Basquete Brasileiro \\
\hline
\end{tabular}

Nota. A fonte foram os próprios autores (2015) 
Durante o processo inicial de estudos bibliográficos, escolha do modelo de avaliação de risco e definição de variáveis, chega-se a um número

de 30 variáveis que após um processo de refinamento inicial diminui-se para 20 variáveis. São Elas:

Tabela 3

Relação das variáveis finais: dimensão comprometimento

\begin{tabular}{l}
\hline Criação de Escritório Comercial no Brasil \\
\hline Realização de jogos entre equipe brasileira e franquia americana no Brasil \\
\hline Tradução do site da NBA.com para o português \\
\hline Abertura de Loja Virtual de produtos oficiais em Português \\
\hline Realização de pelo menos um evento voltado ao consumidor no Brasil de forma regular desde 2003 \\
\hline Realização de Jogos de Pré-temporada entre franquias da NBA no Brasil \\
\hline Realização de jogos entre equipe brasileira e franquia americana nos EUA \\
\hline Parceria Formal de 3 anos com o NBB \\
\hline Acordos de tv que ampliam o número de jogos transmitidos em TV fechada de 2 pra 5 semanalmente \\
\hline Criação de Perfis em Redes Sociais e de conteúdo específico para o público brasileiro. \\
Nota. A fonte foram os próprios autores (2016) \\
Tabela 4 \\
Relação das variáveis finais: dimensão incertezas \\
\hline Economia e Política Brasileira \\
\hline Desenvolvimento do Esporte no Brasil \\
\hline Monopólio Esportivo e Econômico - Futebol no Brasil \\
\hline Cultura Esportiva na Sociedade Brasileira \\
\hline Ídolos Nacionais do Basquete Brasileiro \\
\hline Infraestrutura Esportiva no Brasil - Arenas
\end{tabular}

O processo de criação do índice larNBA, IAR da NBA, se deu a partir da aplicação de questionários a 30 peritos. Os peritos foram convidados a atribuir pesos, considerando a pertinência e atribuir uma nota para as variáveis do questionário, afim de se analisar a realidade do risco de internacionalização da NBA no Brasil em dois momentos: nos anos de 2012 a 2015 e no futuro ano de 2020.

Os questionários foram aplicados via aplicativo Survey Monkey e dirigidos aos peritos em 30 dias via e- mail. Com apenas 25 respostas avançou-se para a criação do índice. Antes da criação do índice submeteuse inicialmente as variáveis a um último refinamento e corte por meio do método da caixa de ferramentas de Godet (2000). Calculou-se a média, o desvio padrão e o coeficiente de variação e procedeu-se com o corte das variáveis não consensuadas, ou seja, com coeficiente de variação igual ou maior que $20 \%$. O resultados deste procedimento estão expostos na tabela e na tabela 5, a seguir.

\begin{tabular}{lcccc}
\hline $\begin{array}{l}\text { Tabela } 5 \\
\text { Corte final de variáveis }\end{array}$ & & & \\
\hline \multicolumn{1}{c}{ Dimensões } & Total & Consensuadas & Total & Consensuadas \\
\hline Incerteza & 10 & 8 & 10 & 5 \\
\hline Comprometimento & 10 & 6 & 10 & 4 \\
\hline Total & 20 & 14 & 20 & 9 \\
\hline
\end{tabular}

Nota. A fonte foram os próprios autores (2016) 
As tabelas 6 e 7 mostram o resultado do processo de corte final de variáveis, onde chegou-se ao número de 14 variáveis no período 2012-2015 de azul claro a 09 variáveis no período 2020, de azul escuro, uma redução esperada que traz mais confiabilidade a pesquisa. Com o número final de variáveis definida, dedicou-se a construção do índice larNBA.

Tabela 6 - Corte de variáveis método de Godet: Período 2012-2015

Fonte Elaborada pelos autores (2016)

\begin{tabular}{|c|c|c|c|c|c|}
\hline & & & Média & $\begin{array}{l}\text { Desvio } \\
\text { Padrão }\end{array}$ & $\begin{array}{l}\text { Coef. de } \\
\text { Variação }\end{array}$ \\
\hline Dimensão & Pilares & Variáveis & & & \\
\hline \multirow{10}{*}{$\begin{array}{l}\overline{2} \\
\text { 男 } \\
\text { 思 } \\
\stackrel{N}{D}\end{array}$} & Institucional & 1. Economia e Política Brasileira & 0,9056 & 0,17 & 19,21 \\
\hline & Mercado & 2. Desenvolvimento do Esporte no Brasil & 0,8656 & 0,15 & 17,74 \\
\hline & Estrutura & $\begin{array}{l}\text { 3. Monopólio Esportivo e Econômico - Futebol no } \\
\text { Brasil }\end{array}$ & 0,5664 & 0,11 & 18,76 \\
\hline & Mercado & 4.Cultura Esportiva na Sociedade Brasileira & 0,4992 & 0,18 & 37,02 \\
\hline & Mercado & 5. Popularidade do Basquete no Brasil & 0,4768 & 0,06 & 12,33 \\
\hline & Mercado & 6. Ídolos Nacionais do Basquete no Brasil & 0,5024 & 0,09 & 18,68 \\
\hline & Estrutura & 7. Infraestrutura Esportiva no Brasil - Arenas & 0,9104 & 0,15 & 16,31 \\
\hline & Mercado & 8. Transmissão de Jogos de Basquete na TV Aberta & 0,56 & 0,09 & 16,37 \\
\hline & Estrutura & 9. Calendário nacional anual de jogos & 0,376 & 0,15 & 40,86 \\
\hline & Estrutura & $\begin{array}{l}\text { 10. Estrutura de Base para a Formação de Novos } \\
\text { Jogadores }\end{array}$ & 0,7424 & 0,11 & 14,51 \\
\hline \multirow{10}{*}{ 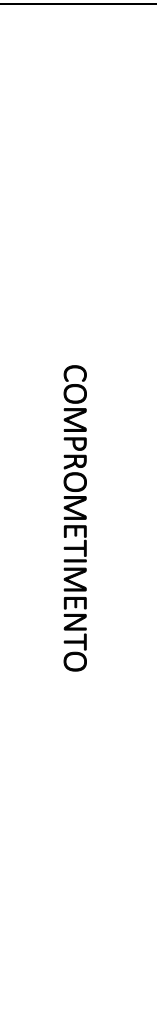 } & Relacionamento & 11. Criação de escritório comercial no Brasil & 0,1712 & 0,02 & 14,34 \\
\hline & Eventos & $\begin{array}{l}\text { 12. Realização de jogos de pré-temporada entre } \\
\text { franquias da NBA no Brasil }\end{array}$ & 0,4352 & 0,08 & 18,06 \\
\hline & Eventos & $\begin{array}{l}\text { 13. Realização de jogos entre equipe brasileira e } \\
\text { franquia americana nos EUA }\end{array}$ & 0,3904 & 0,24 & 0,62 \\
\hline & Eventos & $\begin{array}{l}\text { 14. Realização de jogo entre equipe brasileira e } \\
\text { franquia americana no Brasil }\end{array}$ & 0,4768 & 0,06 & 18,59 \\
\hline & Parcerias & 15. Parceria comercial formal de 3 anos com o NBB & 0,424 & 0,07 & 17,44 \\
\hline & Relacionamento & 16. Tradução do site da NBA.com para o português & 0,0688 & 0,03 & 39,43 \\
\hline & Parcerias & $\begin{array}{l}\text { 17. Acordos de TV que ampliam o número de jogos } \\
\text { transmitidos em TV fechada de } 2 \text { pra } 5 \\
\text { semanalmente aproximadamente }\end{array}$ & 0,48 & 0,07 & 15,59 \\
\hline & Parcerias & $\begin{array}{l}\text { 18. Abertura de Loja virtual de produtos oficias em } \\
\text { português }\end{array}$ & 0,3904 & 0,16 & 41,33 \\
\hline & Eventos & $\begin{array}{l}\text { 19. Realização de pelo menos um evento voltado } \\
\text { ao consumidor brasileiro de forma regular desde } \\
2003\end{array}$ & 0,4448 & 0,06 & 14,53 \\
\hline & Relacionamento & $\begin{array}{l}\text { 20. Criação de Perfis em Redes Sociais e de } \\
\text { conteúdo específico para o público brasileiro }\end{array}$ & 0,0848 & 0,04 & 43,74 \\
\hline
\end{tabular}


Tabela 7 - Corte de variáveis método de Godet: Período 2020

Período 2020 Fonte Elaborada pelos autores (2016)

\begin{tabular}{|c|c|c|c|c|c|}
\hline & & & Média & $\begin{array}{l}\text { Desvio } \\
\text { Padrão }\end{array}$ & $\begin{array}{l}\text { Coef. de } \\
\text { Variação }\end{array}$ \\
\hline Dimensão & Pilares & Variáveis & & & \\
\hline \multirow{10}{*}{\begin{tabular}{l}
$\bar{\sum}$ \\
罗 \\
思 \\
\} & Institucional & 1. Economia e Política Brasileira & 0,872 & 0,16 & 18,59 \\
\hline & Mercado & 2. Desenvolvimento do Esporte no Brasil & 0,8624 & 0,15 & 17,15 \\
\hline & Estrutura & $\begin{array}{l}\text { 3. Monopólio Esportivo e Econômico - Futebol no } \\
\text { Brasil }\end{array}$ & 0,856 & 0,15 & 17,22 \\
\hline & Mercado & 4.Cultura Esportiva na Sociedade Brasileira & 0,2288 & 0,04 & 18,56 \\
\hline & Mercado & 5. Popularidade do Basquete no Brasil & 0,4176 & 0,19 & 45,61 \\
\hline & Mercado & 6. Ídolos Nacionais do Basquete no Brasil & 0,432 & 0,08 & 17,93 \\
\hline & Estrutura & 7. Infraestrutura Esportiva no Brasil - Arenas & 0,6544 & 0,25 & 38,65 \\
\hline & Mercado & 8. Transmissão de Jogos de Basquete na TV Aberta & 0,4768 & 0,26 & 54,42 \\
\hline & Estrutura & 9. Calendário nacional anual de jogos & 0,352 & 0,2 & 56,25 \\
\hline & Estrutura & $\begin{array}{l}\text { 10. Estrutura de Base para a Formação de Novos } \\
\text { Jogadores }\end{array}$ & 0,5424 & 0,27 & 50,2 \\
\hline \multirow{10}{*}{ 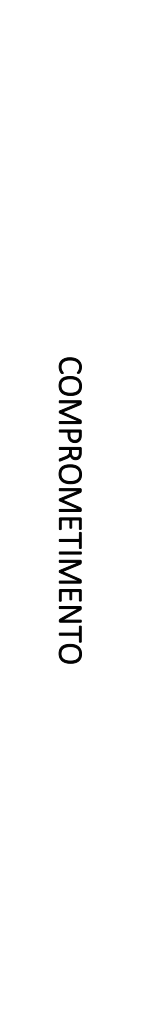 } & Relacionamento & 11. Criação de escritório comercial no Brasil & 0,8128 & 0,11 & 13,38 \\
\hline & Eventos & $\begin{array}{l}\text { 12. Realização de jogos de pré-temporada entre } \\
\text { franquias da NBA no Brasil }\end{array}$ & 0,472 & 0,23 & 48,68 \\
\hline & Eventos & $\begin{array}{l}\text { 13. Realização de jogos entre equipe brasileira e } \\
\text { franquia americana nos EUA }\end{array}$ & 0,3504 & 0,2 & 58,05 \\
\hline & Eventos & $\begin{array}{l}\text { 14. Realização de jogo entre equipe brasileira e } \\
\text { franquia americana no Brasil }\end{array}$ & 0,92 & 0,15 & 16,6 \\
\hline & Parcerias & 15. Parceria comercial formal de 3 anos com o NBB & 0,4336 & 0,24 & 54,23 \\
\hline & Relacionamento & 16. Tradução do site da NBA.com para o português & 0,0768 & 0,01 & 14,42 \\
\hline & Parcerias & $\begin{array}{l}\text { 17. Acordos de TV que ampliam o número de jogos } \\
\text { transmitidos em TV fechada de } 2 \text { pra } 5 \\
\text { semanalmente aproximadamente }\end{array}$ & 0,3744 & 0,25 & 65,49 \\
\hline & Parcerias & $\begin{array}{l}\text { 18. Abertura de Loja virtual de produtos oficias em } \\
\text { português }\end{array}$ & 0,5424 & 0,1 & 19,29 \\
\hline & Eventos & $\begin{array}{l}\text { 19. Realização de pelo menos um evento voltado } \\
\text { ao consumidor brasileiro de forma regular desde } \\
2003\end{array}$ & 0,3696 & 0,28 & 74,61 \\
\hline & Relacionamento & $\begin{array}{l}\text { 20. Criação de Perfis em Redes Sociais e de } \\
\text { conteúdo específico para o público brasileiro }\end{array}$ & 0,2288 & 0,25 & 109,36 \\
\hline
\end{tabular}}
\end{tabular}

A seguir, apresentam-se as etapas para o cálculo do IARnba para se avaliar o grau de internacionalização da NBA no Brasil no período de 2012-2105 e 2020:

Etapa 1: Seleciona-se o número de peritos;

Etapa 2: Encaminham-se os questionários contendo o número de variáveis de avaliação consensuadas, compreendidas nas duas dimensões; no período de 2012-2014 (Incerteza = 8, Comprometimento $=6$ ) e no período de 2020 $($ Incerteza $=5$, Comprometimento $=4)$.

Os peritos serão convidados a avaliar as variáveis aplicando pesos, por meio do grau de pertinência e dar notas ao grau de risco de cada variável nos dois períodos citados acima. 
As variáveis devem ser marcadas em uma escala Likert de 1 a 5, em ambas as formas de avaliar, onde atribui-se a pesos a escala: 1 =irrelevante; 2 = pouco pertinente; $3=$ medianamente pertinente; $4=$ pertinente; 5 = muito pertinente e as notas $1=$ não representa risco; 2 = risco pequeno; 3 = risco mediano; 4 = risco considerável; 5 = risco alto.
Etapa 3: Colhem-se os questionários respondidos dos peritos conforme seus julgamentos em cada variável;

Etapa 4: Calcula-se o índice IARnba. A aplicação da fórmula deve ser realizada de acordo com cada um das dimensões incerteza e comprometimento. Onde IARnba = (IARi, IARc) que são calculados pela fórmula abaixo:

$$
I A R i=\frac{\sum_{i=1}^{N_{e s p}} \frac{x_{p i} * x_{n i}}{25 * N_{e s p}}}{N_{v i}}
$$

Na qual:

IARi: Índice de Análise de Risco de Incerteza;

IARc: Índice de Análise de Risco de Comprometimento;

Xpi: Média de Pesos de Incerteza;

Xni: Média de Notas de Incerteza;

Xpc: Média de Pesos de Comprometimento;

Xnc: Média de Notas de Comprometimento;

25: Produto dos valores máximos de Pesos e Notas;

$\mathrm{N}_{\text {esp }} \mathrm{N} i$ : Número de peritos;

Nvc: Número de Variáveis Consensuadas de Comprometimento;

$$
I A R c=\frac{\sum_{i=1}^{N_{e s p}} \frac{x_{p c} * x_{n c}}{25 * N_{e s p}}}{N_{v c}}
$$

$\mathrm{N}_{\text {vi: }}$ Número de Variáveis Consensuadas de Incerteza.

Inicialmente calcula-se a soma do produto das médias obtidas pelos pesos Xp e notas $X n$ atribuídas pelos peritos dividido por seu peso máximo atribuído, 25, multiplicado pelo número de peritos (Nesp).

Depois divide-se pelo número de variáveis consensuadas de cada dimensão Nv. Logo depois calcula-se o índice para os dois períodos estudados: 2012-2015 e 2020.

Definido o índice larNBA, partiu-se para a análise do risco de internacionalização da NBA no Brasil, considerando o período atual 2012-2015 e o período de 2020. Aplicando-se a formula, o resultado denota a situação retratada abaixo:

\section{Tabela 8}

Dados da avaliação de risco de internacionalização

\begin{tabular}{ccc} 
& Situação Atual & Situação Futura \\
\hline IARc & $2012-2105$ & 2020 \\
\hline IARi & 0,41 & 0,59 \\
\hline IARnba & 0,69 & 0,65 \\
\hline$(0,41 ; 0,69)$ & $(0,59 ; 0,65)$
\end{tabular}

Nota. A fonte foi o próprio autor (2016)

O grau de internacionalização da NBA no Brasil, conforme demonstrado na figura 3 abaixo é interpretado de acordo com a proposta de Figueira de Lemos, Johanson, Vahlne; (2011) que denota uma tendência geral de Risco Decrescente. 


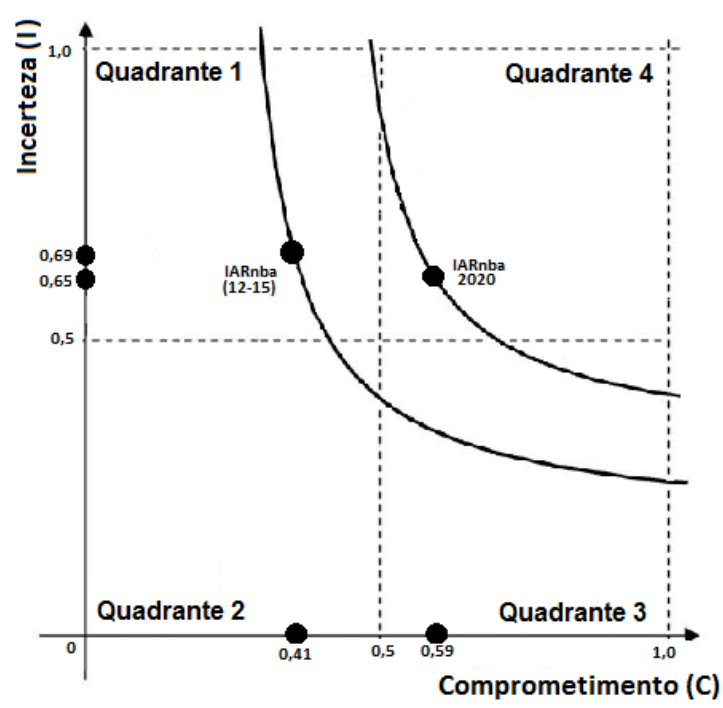

Figura 3. Internacionalização da National Basketball Association (NBA) no Brasil Fonte: Elaborada pelo autor (2016).

Durante os 5 anos estudados as incertezas tendem a diminuir e o comprometimento tende a aumentar, diminuindo o risco. Nos próximos parágrafos examinaremos os dois momentos isolados.

\section{a) Período 2012-2015}

Todo estágio inicial de internacionalização, quando uma firma entra em um determinado mercado, ela assume um risco inicial de entrada neste mercado.

Este risco é determinado pelo aporte inicial de recursos (comprometimento) necessários a entrada e pelo nível de incerteza (conhecimento) que aquela empresa tem daquele mercado (FIGUEIRA DE LEMOS; JOHANSON; \& VAHLNE, 2011).

Esse níveis iniciais são retratados pelos dados pesquisados no período de 2012-2015. A figura aponta o índice IARnba marcado pelo $(0,41 ; 0,69)$, com nível de incerteza inicialmente retratadas acima da metade, IARi = 0,69 e nível de comprometimento menor que a metade, $I A R C=0,41$.

Adaptando a metodologia proposta por Figueira de Lemos, Johanson, e Vahlne (2011), a figura demonstra que a NBA entre 2012 e 2015 posicionase no quadrante 1 . Alta incerteza e baixo comprometimento retrata a fase inicial de internacionalização, porém tende a evoluir para uma situação onde já se detém um conhecimento inicial suficiente do mercado brasileiro, a ponto de se permitir a instalação de um escritório no país. Este nível permite conexões com parceiros de mercado e fechamento de contratos estratégicos de curto prazo, que não envolvam um comprometimento alto (Figueira-de-lemos, Johanson, \& Vahlne, 2011).

Os níveis de incerteza expostos inicialmente refletem que apesar da NBA manter um relacionamento de mais de 10 anos com o Brasil, este conhecimento prévio não foi suficiente para o nível de incerteza diminuir. Sobre esta afirmação, Arnon de Mello, diretor executivo da NBA no Brasil em entrevista ao portal UOL (2016), afirma que a questão econômica brasileira tem afetado um crescimento maior da NBA no Brasil nos últimos dois anos, como todos os produtos da liga são atrelados ao dólar, perdeu-se em competitividade e o crescimento foi mais lento que o esperado.

b) Período 2020

No segundo período da pesquisa, onde foi retratado o cenário futuro em 2020, a figura demonstra que a NBA se encaminhará para os quadrantes 3 e 4 , passando por uma situação de Comprometimento cada vez maior e uma queda do nível de Incerteza.

A figura aponta o índice IARnba marcado pelo $(0,59 ; 0,65)$, com nível de incerteza menor que o retratado no período anterior, marcando, IARi $=0,65$ e nível de comprometimento um pouco maior, já acima da metade, IARc = 0,59.

A visão dos peritos é que em 2020 a NBA se encontrará com relações sólidas com o mercado e público Brasileiros. Investimentos maiores e crescentes demonstram segurança da empresa no 
conhecimento do mercado. Novamente Arnon de Mello em entrevista ao UOL (2016) ressalta que "é preciso ter paciência no Brasil, a NBA sabe que o ciclo é longo, nada acontece da noite para o dia", realidade expressa na figura 3.

De acordo com Figueira de Lemos, Johanson, e Vahlne (2011) o nível de incerteza vem baixando, porém de forma ainda muito lenta. A velocidade com que se implementa coisas no Brasil é menor que em outros cantos do mundo, o conhecimento maior do mercado vai fazer com que o nível de incerteza seja menor, porém não tão rápido quanto poderia ser.

De acordo com o UOL (2016), a NBA investe em um projeto a longo prazo no Brasil, chamado Jr. NBA. Que visa levar a prática do basquete a crianças carentes com a clara intenção de desenvolver o esporte no país. Em 2020 estes e outros projetos já devam estar gerando lucros para a liga.

c) Explicações para as possíveis relações Incerteza $\times$ Comprometimento

Em análise comparativa aos resultados dos dois momentos estudados 2012-2015 e 2020, pode ser observado nas subseções acima que o Risco decresceu no período estudado. Considerando que Figueira de Lemos, Johanson, e Vahlne (2011) afirmam que $\mathrm{R}=\mathrm{C}^{*} \mid$ e que quando $\mathrm{O}$ comprometimento aumenta gera mais conhecimento do mercado o que ocasiona diminuição da Incerteza e diminuição do risco, percebe-se um aumento dos valores de IARc e diminuição dos valores de IARi no período de 2020 onde caracteriza-se a diminuição do Risco.

Porém a relação entre o ganho de comprometimento, IARc, comparando-se os dois períodos em questão, mostra-se desproporcional a perda do nível de Incerteza, IARi, a ponto de gerar questionamentos no estudo.

Credita-se esta desproporcionalidade à instabilidade do Mercado Brasileiro. O modelo proposto por Figueira de Lemos, Johanson, e Vahlne (2011) utilizado neste trabalho considera a aplicação do modelo a mercados estáveis.

O Brasil hoje é considerado um mercado não estável com problemas de ordem econômica e política como fatores que afetam a credibilidade de suas instituições (BLOOMBERG, 2016).

A interpretação é de que os peritos consultados neste trabalho consideram que a NBA Brasil terá em
2020 o conhecimento suficiente do mercado brasileiro a ponto de permitir investimentos maiores, gerando um comprometimento maior mesmo em um ambiente não estável onde o nível de incerteza não diminua tanto quanto esperado.

Alguns motivos podem ser enumerados no sentido de reforçar este pensamento:

a) O produto é superior. A NBA é reconhecida no mundo todo pelo sua excelência em serviços prestados. A organização de seus jogos trazem uma atmosfera única apelidada de "Estilo NBA" e elevam um simples jogo de basquete a condição de um espetáculo de entretenimento (PRONI, 1998);

b) O histórico de sucesso na Internacionalização de suas operações, o Brasil é o 15 escritório da NBA no mundo (PORTUGAL NETO \& FORTE, 2015), casos de sucesso como o da internacionalização na China (BLOOMBERG, 2007), demonstram que o modus operandi da NBA é trabalho a longo prazo;

c) O Basquete retomou o histórico de esporte popular no Brasil. As conquistas recentes de organização e estruturação voltaram a gerar interesse do público pelo esporte. A quantidade crescente de atletas brasileiros nas franquias da NBA é outro indicio disso (IBOPE REPUCON, 2015).

\section{CONCLUSÃO}

O resultado da pesquisa demonstrada neste trabalho, contribuí de forma relevante no entendimento do processo de Internacionalização de uma organização esportiva em busca de novos mercados pelo mundo e os riscos envolvidos no processo. Ajudará a simular e minimizar movimentos estratégicos de organizações esportivas em busca de novos mercados mundo afora.

Quanto ao primeiro objetivo específico proposto o Índice IARnba é calculado por meio de um sistema de avaliação de variáveis ligadas as dimensões Incerteza, Índice IARi e Comprometimento, Índice IARc que juntos definem o IARnba= (IARc, IARi), sendo aplicável a qualquer mercado e/ou organização esportiva que se queira trabalhar, dado que é ancorado em fatores

Quanto ao segundo objetivo específico proposto à classificação do Risco de Internacionalização da NBA no Brasil, demonstrou que em cinco anos, 20152020, haverá possivelmente uma diminuição do risco de internacionalização da NBA no Brasil, e que o período gerará um aprofundamento e fortalecimento 
nas relações com o mercado e com diminuição de incertezas, o que permitirá, caso necessário, um recuo em investimentos mais comprometedores dado a instabilidade do mercado brasileiro.

Quanto à contribuição gerencial e de ordem prática, a pesquisa mostrou uma fotografia do atual estágio da Internacionalização da NBA no Brasil, permitindo comparar de forma prática o impacto e a importância de ações realizadas na construção desse processo, mapeando todos os movimentos dentro desse processo nos últimos 20 anos.

Dentre as limitações encontradas nesta pesquisa, destaca-se a indisponibilidade de alguns especialistas em responder o questionário desenvolvido; pois acredita-se que, quanto mais respostas obtidas, mais apuradas seriam as notas atribuídas aos riscos das variáveis, por exemplo. A grande quantidade de fontes não acadêmicas, como revistas, jornais e sites

\section{REFERÊNCIAS}

Balassiano, F. (2015) Bala na Cesta - Arnon de Mello fala dos primeiros passos da parceria entre NBA e NBB. Recuperado de: http://balanacesta.blogosfera.uol.com.br/2015/03/1 o/arnon-de-mello-fala-dos-primeiros-passos-daparceria-entre-nba-e-nbb/

Balmer, J. M. \& Gray, E. R. (2003) Corporate brands: what are they? What of them? European Journal of Marketing, 37(7-8), 972-997.

Bauer, H. H.; Sauer, N. E. \& Schmitt, P. (2005) Customer-based brand equity in the team sport industry. European Journal of Marketing, 39, 496513.

Bloomberg. (2007). A Slam Dunk for NBA in China. Recuperado de: http://www.bloomberg.com/news/articles/2007-1023/a-slam-dunk-for-the-nba-in-chinabusinessweekbusiness-news-stock-market-and-financial-advice

$$
\text { (2016). O Brasil ainda é o pais do }
$$
futuro. Recuperado de: https://www.bloomberg.com.br/blog/o-brasil-aindae-o-pais-futuro/

Buckley, P. J. \& Casson, M. (1976), The future of the multinational enterprise. London: Macmillan, v. 1.

Chadwick, S. (2009) From outside lane to inside track: sport management research in the twenty-first century. Management Decision, 47, 191-203. de internet; utilizadas neste estudo para justificar ações e eventos realizados pela NBA evidencia a limitada quantidade de material disponível na academia o que acaba por tornar o trabalho menos científico e pode ser encarado como limitação.

Para pesquisas futuras, sugere-se para efeito de complemento de informações neste estudo, a aplicação de análise de cenários sobre a atuação da NBA no Brasil para os próximos 5 ou 10 anos, a ampliação do estudo dentro da área de internacionalização de ligas esportivas tomando, por exemplo, a atuação na NBA em outros países em desenvolvimento nos chamados BRICS (China, Rússia, África do Sul e Índia) como também a aplicação desta metodologia a outras ligas e esportes como a NFL, liga americana de futebol americano, a WSL, liga mundial de surf e a UFC, liga mundial de MMA.

Child, J. \& Rodrigues, S. B. (2007) The role of trust in international entrepreneurship. In: ENCONTRO ANUAL DA ASSOCIAÇÃO NACIONAL DOS PROGRAMAS DE PÓSGRADUAÇÃO EM ADMINISTRAÇÃO, 31., Rio de Janeiro. Anais. Rio de Janeiro: ANPAD, CD-ROM.

Deloitte Brasil. (2010) Brasil, Bola da vez: Negócios e investimentos a caminho dos mega eventos esportivos. Recuperado de: http://www.deloitte.com/assets/DcomBrazil/Local\% 20Assets/Documents/Estudos\%20e\%20pesquisas/Pe squisa\%20Brasil\%20bola\%20da\%20vez\%20\%20Deloi tte\%20e\%20IBRI.PDF

Dikmen, I. \& Birgonul, M. T. (2006) An analytic hierarchy process based model for risk and opportunity assessment of international construction projects. Canadian Journal of Civil Engineering, 33(1), 58-68.

Dunning, J. H. (2006) Towards a new paradigm of development: implications for the determinants of international business. Transnational corporations, 15(1), 173-227.

Eisenberg, D. (2003) The NBA's global game plan. Time Europe, 161(13), 115-118.

Euchner, C. H. D. (2013) The American: A Magazine of Ideas. Recuperado de: <http://www.american.com/archive/2008/januaryfebruary-magazinecontents/hoop-dreams> 
Figueira-de-lemos, F.; Johanson, J. \& Vahlne, J. E. (2011) Risk management in the internationalization process of the firm: A note on the Uppsala model. Journal of World Business, 46(2), 143-153.

Forbes. (2018). The World's 50 Most Valuable Sports Teams. Recuperado de: < https://www.forbes.com/sites/kurtbadenhausen/20 18/07/18/the-worlds-most-valuable-sports-teams$\underline{2018 />}$

Forlani, D.; Parthasaranthy, M. \& Keaveney, S. M. (2008) Managerial risk perceptions of international entry-mode strategies. The interaction effect of control and capability. International Marketing Review, 25(3), 292-311.

Freitas, H.; Oliveira, M.; Saccoi, A. Z.\& Mascarola, J. (2000) O método de pesquisa survey. Revista de Administração, São Paulo, 35(3), 105-112.

Geleilate, J. M. G. (2011) O Processo de Internacionalização dos Bancos Brasileiros: motivações e estratégias. 269 p. Dissertação (Mestrado em Administração de Empresas) Universidade de Fortaleza (UNIFOR), CMA, Fortaleza.

Godet, M. (2000) A caixa de ferramentas da prospectiva estratégica: problemas e métodos. Caderno do Centro de Estudos de Prospectiva e Estratégia, Lisboa, n. 5.

Goldman, M. \& Johns, K. (2009) Sportainment: Changing the pace of limited-overs cricket in South Africa. Management Decision, 47(1), 124-136.

Goulart, L.; Brasil, H. V.; Arruda, C. A. (1996) A internacionalização de empresas brasileiras: motivações e alternativas. Internacionalização de empresas brasileiras. Rio de Janeiro: Qualitymark.

Howard, D. R. \& Crompton, J. L. (2004) Financing sport. 2nd ed. Morgantown, WV: Fitness Information Technology.

Ibope Repucom. (2015) 39\% dos superfãs de basquete do Brasil acompanham a NBA, aponta pesquisa do IBOPE Repucom. 2015. Recuperado de: $<$ http://www.iboperepucom.com/br/noticias/39dos-superfas-de-basquete-do-brasil-acompanham-anba-aponta-pesquisa-do-ibope-repucom-2/>

Javalgi, R. R. G., Gross, A. C., Benoy Joseph, W., \& Granot, E. (2001) Assessing competitive advantage of emerging markets in knowledge intensive business services. Journal of Business \& Industrial Marketing, 26(3), 171-180.

Johanson, J. \& Vahlne, J. (1977) The internationalization process of the firm: a model of knowledge development and increasing market commitment. Journal of International Business Studies, 8, 23-32.

(2009) The Uppsala internationalization process model revisited: From liability of foreignness to liability of outsidership. Journal of International Business Studies, 40(9), 1411-1431).

Kian, E. T. (2009) The NBA: Not Yet a Global Sport League in Newspaper Coverage. The SMART Journal.

Lumpkin, G. T. \& Dess, G. G. (1996) Clarifying the entrepreneurial orientation construct and linking it to performance. Academy of Management Review, 21(1), 135-172.

Mcdougall, P. P. (1989) International versus domestic entrepreneurship: new venture strategic behavior and industry structure. Journal of Business Venturing, 4(6), 387-400.

NBA. (2018) National Basketball Association. 2015. Recuperado de: <http://www.nba.com>.

Pluri Consultoria. (2014) O PIB do Esporte Brasileiro. Recuperado de: $<$ http://new.pluriconsultoria.com.br/wpcontent/uploads/2014/11/PIB-Esporte.pdf>.

Portugal Neto, A. F.; Forte, S. H. A. C. (2015) Internationalization process of the NBA in Brazil: differentiation, barriers and entry modes. In: EIBA CONFERENCE, 41., 2015, Rio de Janeiro. 2015. Rio de Janeiro: EIBA, 2015. 1-16.

Proni, M. W. (1998) Marketing e organização esportiva: elementos para uma história recente do esporte-espetáculo. Conexões, 1(1), 82-94.

Raisanen, J. A. S. (2003) Evolution of internationalization theories related to the born global concept. Helsinki: Helsinki University of Technology,

Ratten, V. (2018) The Sport Industry and Entrepreneurship. In: Sport Entrepreneurship. Management for Professionals. Springer, Cham.

Ratten, V. (2011a) International sport management: current trends and future 
development. Thunderbird International Business Review, 53(6), 679-686.

. (2011b) Practical Implications and Future Research Directions for International Sport Management. Thunderbird International Business Review, 53(6), 763-770.

Sharma, V. M. \& Erramilli, M. K. (2004) Resourcebased explanation of entry mode choice. Journal of Marketing Theory and Practice, 1-18.

Schweizer, R.; Vahlne, J. \& Johanson J. (2010) Internationalization as an entrepreneurial process. Journal of International Entrepreneurship.
Sport+Markt. (2011) Estudo sobre Prática de Esportes. Rio de Janeiro: Sport+Markt Brasil.

UOL. (2016) NBA prepara volta ao Brasil em 2017 e avalia arena em Fortaleza e Curitiba -Recuperado de: https://esporte.uol.com.br/basquete/ultimasnoticias/2016/09/20/nba-prepara-volta-ao-brasilem-2017-e-avalia-arena-em-fortaleza-e-curitiba.htm

Williamson, O. E. (1995) Transaction cost economics and organization theory. In: Williamson, O. E. (Ed.). Organization theory from Chester Barnard to the present and beyond. New York: Oxford University Press.

\title{
Sobre os autores:
}

Amaury Floriano Portugal Neto - Centro Universitário Inta - Sobral, Ceará, (Brasil). E-mail: amauryportuga@gmail.com Orcid id: https://orcid.org/0000-0002-3531-1509

Sergio Henrique Arruda Cavalcante Forte - Universidade de Fortaleza - INIFOR, Fortaleza, Ceará, (Brasil). E-mail: sergioforte@unifor.br Orcid id: https://orcid.org/0000-0002-8406-0910

\section{RISK ASSESSMENT IN THE PROCESS OF INTERNATIONALIZATION OF THE NBA IN BRAZIL}

\author{
Amaury Floriano Portugal Neto, Sergio Henrique Arruda Cavalcante Forte \\ Centro Universitário INTA, Sobral, Ceará, (Brasil) \\ Universidade de Fortaleza - INIFOR, Fortaleza, Ceará, (Brasil)
}

\section{ARTICLE DETAILS}

Article history:

Received: 16 November 2018

Accepted: 05 September 2019

Available online January: 01 th 2020

Double Blind Review System

Scientific Editor

Ilan Avrichir

\section{Key words}

Internationalization process

Risk assessment

Management of the sport

Basketball

NBA

\section{RESUMO}

Objective: To evaluate the risks involved in the process of internationalization of the NBA in Brazil in its initial period, 2012-2015, and a projection on your future 5 years ahead, 2020.

Method: The methodology included six stages, involving bibliographical research, consultation of experts for the survey of drawing, evaluation and refinement of relevant variables, allowing the formatting of the index of Risk Assessment of the NBA (IARnba) and its application in the light of the model of Figueira de Lemos, Johanson and Vahlne (2011) in the analysis of the internationalization of the NBA, having the variables of (C) commitment and (I) Uncertainty interpreted

Main Results: The study showed that during the two periods studied the risk of internationalization of the NBA in Brazil tends to decrease, despite the levels of uncertainty remain in a small fall. The increasing level of knowledge of the brazilian market followed by investments that indicate a greater commitment corroborates this view.

Relevance/originality: Its relevance is due to its originality in portraying the focus on internationalization of sports leagues in emerging countries and unstable economy like Brazil.

Theoretical/methodological contributions: its contribution to the literature addressing the topic of sports business in the light of the theory of the internationalization of the firm, portraying the search for international markets for sports leagues in the world and to demonstrate and evaluate the risks of these movements by means of an index. 


\title{
EVALUACIÓN DE RIESGOS EN EL PROCESO DE INTERNACIONALIZACIÓN DE LA NBA EN BRASIL
}

\author{
Amaury Floriano Portugal Neto, Sergio Henrique Arruda Cavalcante Forte \\ Centro Universitário INTA, Sobral, Ceará, (Brasil) \\ Universidade de Fortaleza - INIFOR, Fortaleza, Ceará, (Brasil)
}

\section{DETALLES DEL ARTÍCULO}

\section{Historia del Artículo:}

Recibido: 16 noviembre 2018

Aceptado: 05 septiembre 2019

Disponible en línea: 01 de enero 2020

\section{Double Blind Review System}

Editor Científico

llan Avrichir

\section{Palabras-clave:}

Proceso de internacionalización

La evaluación del riesgo

Gestión del deporte

El baloncesto

NBA

\section{RESUMO}

Objetivo: evaluar los riesgos involucrados en el proceso de internacionalización de la NBA en Brasil en su período inicial, 2012-2015, y una proyección sobre su futuro 5 años venideros, 2020

Método: La metodología incluyó seis etapas, que implican la investigación bibliográfica, la consulta de expertos para el estudio de la elaboración, evaluación y refinamiento de las variables relevantes, permitiendo que el formato del índice de evaluación de riesgos de la NBA (IARnba) y su aplicación a la luz del modelo de Figueira de Lemos, Johanson y Vahlne (2011) en el análisis de la internacionalización de la NBA, con las variables de compromiso (C) e (I) Incertidumbre interpretado.

Principales resultados: El estudio demostró que, durante los dos períodos estudiados el riesgo de la internacionalización de la NBA en Brasil tiende a disminuir, a pesar de que los niveles de incertidumbre permanecen en una pequeña caída. El aumento del nivel de conocimiento del mercado brasileño, seguido por las inversiones que indican un mayor deterioro corrobora esta opinión.

Pertinencia y originalidad: Su importancia se debe a su originalidad en retratar el foco en la internacionalización de las ligas deportivas en países emergentes y la inestabilidad de la economía como Brasil.

Teórica/contribuciones metodológicas: su contribución a la literatura aborda el tema del negocio del deporte a la luz de la teoría de la internacionalización de la empresa, describiendo la búsqueda de mercados internacionales de las ligas deportivas en el mundo y para demostrar y evaluar los riesgos de estos movimientos por medio de un índice.

\section{Para citar este artigo:}

Portugal Neto, A., \& Forte, S. (2020). Avaliação de Risco no Processo de Internacionalização da NBA No Brasil. Internext, 15(1), 71-87. doi: http://dx.doi.org/10.18568/internext.v15i1.480 